\title{
PROCESSO DE TRANSIÇÃO DO FAMILIAR PARA O PAPEL DE CUIDADOR FAMILIAR DE UM IDOSO DEPENDENTE
}

\author{
Samara Gonçalves de Oliveira; Universidade do Estado do Rio de Janeiro; \\ samarasog@gmail.com
}

Célia Pereira Caldas; Universidade do Estado do Rio de Janeiro; celpcaldas@hotmail.com

\section{RESUMO}

Introdução: a transição do familiar para o papel de cuidador de um idoso dependente é vista na Teoria das Transições de Afaf Meleis como uma transição situacional por ser um processo complexo que envolve inúmeras variáveis. O objetivo deste estudo é identificar os condicionantes, facilitadores e inibidores na transição de familiares para o papel de cuidadores familiares de um idoso dependente no domicílio e as intervenções de enfermagem necessárias para uma transição saudável. Método: trata-se de uma revisão realizada nas bases de dados Medical Literature Analysis and Retrieval System onLine (MEDLINE) via PubMed, Latino-Americana e do Caribe em Ciências da Saúde (LILACS) e CINAHL. A estratégia de busca utilizada foi a seguinte: operador booleano And, recorte temporal 2015-2019, idiomas português, inglês e espanhol e descritores: "aged", "family" e "Home Nursing". Resultados: Após a avaliação, 23 artigos foram selecionados por atenderem a questão de pesquisa e 374 excluídos. Os resultados da pesquisa, foram organizados em três categorias: "condicionantes inibidores da transição saudável ao tornar-se cuidador de um idoso dependente", "condicionantes facilitadores da transição saudável ao tornar-se cuidador de um idoso dependente" e; "intervenções terapêuticas de enfermagem para uma transição saudável". Conclusão: o processo de transição de um familiar para o papel de cuidador familiar de um idoso dependente é extremamente complexo. Requer suporte estruturado, para que o período de instabilidade não perdure. Com as intervenções terapêuticas de enfermagem é possível para o familiar, alcançar a maestria da transição e a estabilidade.

Palavras-chave: Idoso; Família; "Assistência domiciliar".

Agradecimentos: À FAPERJ - Fundação de Amparo à Pesquisa do Estado do Rio de Janeiro pelo apoio financeiro essencial para realização dessa pesquisa. 